\title{
Bas-relief Layout Arrangement via Automatic Method Optimization
}

\begin{abstract}
It is significant to achieve automatic arrangement for bas-relief layout which can be noticeably more efficient than the time-consuming manual process. In fact, nearly none work has been reported in terms of bas-relief layout arrangement. In this paper, we propose a novel approach to tackle this problem. Specifically, we first identify the evaluation indicators to account for different aesthetic factors, and model the goodness of each indicator. We then cast the bas-relief layout as a combinatorial optimization problem based on those evaluation indicators and a geometric mean model. The contribution of this paper is to propose an objective function for bas-relief layout and apply simulated annealing algorithm for optimization. Experiments show that our method is effective, in terms of layout arrangement for bas-relief generation. In addition, this method can synthesize a few models arrangement and investigate which evaluation indicators will affect the aesthetic perception of the bas-relief.
\end{abstract}

Keywords: Bas-relief layout, Simulated annealing, Combinatorial optimization problem

\section{Introduction}

Sculpture, an important part of culture, has been widely used for thousands of years. Bas-relief is a representative type of sculpture in which carved figures slightly emerge from a background. Compared with other types of sculpture, the prominent features of basrelief are a spatial structure carved into a very narrow depth range and two-dimensional or planar characteristics. The length proportion of figures in bas-relief is maintained and the contour shapes are approximately painted. Therefore, bas-relief can deal with the spatial relation according to the principle of painting.

With the emerging of digital bas-relief [1], the production of bas-relief requires less imagination and practical skills of sculptors. Recent advances in digital relief generation such as 3D based geometric modeling

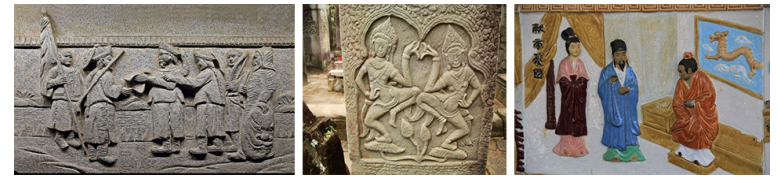

(a)
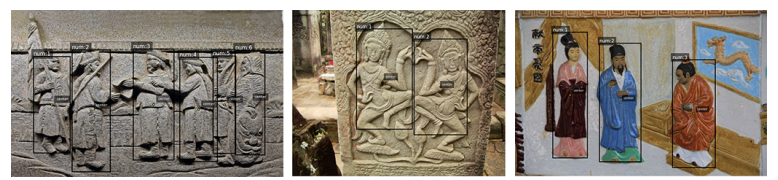

(b)

Figure 1: (a) Bas-relief sculptures with multiple characters in a good manner and harmonious layout in one scene. (b) Manually labeled bas-relief dataset for this work.

overcame many difficulties. However, there are still many challenges regarding the automatic generation of bas-relief with a high aesthetic value. For example, it is fairly arduous and hard to arrange different objects reasonably in a certain area, as shown in Figure 1(a). With recent popularity of 3D printing and personalized customization, procedural methods for an automated relief layout are demanded since it would be tedious and time-consuming to manually model massive multimodel relief. The aesthetic and creative product of basrelief would be best designed by professional sculptors, but manual bas-relief producting is difficult and timeconsuming. Motivated by the above, we propose a novel approach that can automatically generate bas-relief for model layout arrangement by taking important aesthetic factors into account. The approach that we present in this paper achieves this goal in two steps. First, we identify the evaluation indicators of the bas-relief arrangement on top of the photographic composition, literature and valuable bas-relief work. This step is performed only once in advance, acting as a pre-processing. As shown in the example in Figure 1(b), we visualize these evaluation indicators which are collected by manually labeling the relief dataset.

Given an arbitrary initial layout, optimizing it sub- 
ject to human factors is not an easy task, because the search space can be prohibitively large. To address this issue, in the second step the initial layout is adjusted iteratively by minimizing an objective function that accounts for the evaluation indicators, such as height difference, location of bounding rectangle, curvature and so on, wherein the goodness functions of the indicators are encoded as terms in the objective. In the optImization process, in order to ensure the robusness of the algorithm, the ability to optimize complex nonlinearities, and the characteristics fewer initial constraints. We optimize the overall objective function using simulated annealing, which is formulated with a weighted geometric mean combined model. To our knowledge, this is the first work of automatic layout optimization for bas-relief generation. Experiments demonstrate that our approach is capable of generating quality bas-relief with optimized layout.

\section{Related Work}

\subsection{Relief Generation}

Bas-relief generation has been an attractive topic in the computer graphics community for over two decades. Recently, a digitial relief review work can be found in [2]. It divides the methods into image modeling and 3D model modeling. The related work in this paper will be introduced from 3D model modeling.

To preserve detailed features in the bas-relief and achieve real-time processing with high efficiency, Zhang et al.[3] presented a different bas-relief generation algorithm based on geometric compression starting from a 3D mesh input. Ji et al.[4] presented a novel method for digital bas-relief modeling with intuitive style control, which adopted the sparse linear system to solve the bas-relief modeling and stylization simultaneously. Ji et al.[5] proposed a two-scale bas-relief modeling method, which was computationally efficient and easily produced different styles of bas-relief with fine details in real time. Wang et al. Wang et al.[6] proposed a modeling method of relief based on visual attention mechanism and transfer learning, which uses semantic neural network to learn texture and structure representation, and generates new texture images on the basis of preserving the richness of relief details.

[7] articulated a novel 3D surface modeling, featurecentric Empirical Mode Decomposition (EMD). The key idea was to explicitly formulate details as an oscillation between local minima and maxima. [8] developed an interactive modeling system for complex geometric detail transformation, which was also based on EMD on multi-scale 3D shapes. The method took full advan- tage of the multi-scale representation and was computed via EMD which made the algorithm much more flexible. The aforementioned methods can achieve excellent results in terms of preserving the details of bas-relief. Despite this, the research on improving the aesthetics of bas-relief techniques in the field of surface bas-relief generation has been rarely explored.

Given the current methods, Hu et al. [9] presented a method to generate digital bas-relief with the consideration of placing the image at the optional position along the shape. [10] presented a novel approach for bas-relief generation and synthesis, which integrated the visual saliency and photographic composition rules. Their approach was able to effectively produce bas-relief with a reasonable layout and distinct details. Ji et al.[11] proposed a bas-relief generation method that expanded the bas-relief shape space and generated diversified styles of bas-relief. The most prominent advantage of the method is that it transfers details from one region to others.Ji et al. [12] designed an end-to-end depth neural network for scene relief modeling. This method takes the model height field as the input, extracts the detail features of different scales through convolution network, and finally generates high-quality relief model.

\subsection{Object Arrangement}

In the category of practical applications of 3D object arrangement, furniture arrangement is an important research topic in computer graphics. According to the recent furniture layout review[13], the layout problem requires a lot of prior knowledge to select reasonable objects and arrange objects appropriately. The existing works on $3 \mathrm{D}$ object synthesis have mainly solved two subproblems. One is selecting a proper subset of furniture objects[14, 15, 16], and the other is placing them into a given room with a proper layout $[17,18]$. This is similar to our objective; that is distributing 3D models into a proper bas-relief layout.

To enrich a 3D indoor scene with small objects, $\mathrm{Fu}$ et al. [19] presented a user-guided 3D indoor scene enrichment framework to help users effectively apply their rules in small objects arrangement. They learned statistical information from image datasets and constructed an objective function that considered constraints and arrangement rules specified by users. Kan et al. [20] presented a novel method for automated interior design based on a genetic algorithm. They additionally designed a system that automatically populated indoor virtual scenes with furniture objects, whose positions and orientations were optimized according to aesthetic, ergonomic and functional rules (i.e., interior design guidelines). These guidelines were represented as math- 
ematical expressions which formed the objective function. yang et al. [21] presents a method to automatically extract and reconstruct 3D geometry of indoor scene from a single 2D panorama. The sampled perspective sub view is used to extract geometric cues and semantic cues for ground level estimatio.

In our method, we also present some evaluation indicators that have relevance to aesthetic and composition rules. Then we formulate these evaluation indicators mathematically and integrate them into an objective function. Finally, we optimize the initial relief positions by solving the objective function.

\section{Overview}

We propose a bas-relief layout technique that can automatically place one or two 3D objects at desired positions. Our system takes 3D source objects and a target model as inputs. We assume that the mapping region, which is the source models' target zone, is already given. Our goal is to automatically generate a suitable and aesthetic layout for all source models on the relief.

Our core idea is to determine whether a specific sculpture for the input relief follows the defined rules of artistic aesthetics. Inspired by furniture arrangement techniques, we present multiple factors which describe the layout, and extract them as evaluation indicators for the layout process to follow. To obtain the measurements, we use statistical methods for different values of each evaluation indicator in the bas-relief dataset.

The mapping space of our problem could be irregular and highly complex, and the relief layout depends on numerous factors. Because factors that affect the layout could be interdependent in the optimization process, it is very difficult to deduce a global optimization scheme or closed-form solution that yields a unique optimum [18]. To overcome this problem, we use a stochastic optimization method, specifically simulated annealing with the Metropolis criterion [22], to search for a good approximation to the optimum. To determine a good approximation in a reasonably short time, we present a weighted geometric means combination forecasting model. Note that the evaluation of a relief can be subjective though. Hence, after we generate the final bas-relief results with the arrangement, we perform a perceptual study to validate the synthesized results. Figure 2 illustrates the overview of our bas-relief generation method.

\section{Evaluation Indicator Extraction}

First, we collect 200 images of bas-relief layout from the Internet to build a dataset. Then, based on pho- tographic composition rules, the literature and certain evaluation indicators, we extract the evaluation indicators that can affect the bas-relief layout. In particular, we first describe a set of model-dependent evaluation indicators, and combine them to form the overall relief layout standard. We next adopt the LabelImg tool to mark the bounding box of each bas-relief model in the image dataset. From the generated json file which contains the $x_{\min }, y_{\min }, x_{\max }, y_{\max }$ coordinates of each bas-relief model image, we calculate the distribution of each type of evaluation indicators. We finally fit the goodness functions of each evaluation indicator.

\subsection{Evaluation Indicators of Bas-relief Layout}

As reviewed in Section 2, the literature offers many evaluation indicators, such as curvature of relief and projected area, which may contribute to the overall aesthetic arrangement. However, previous attempts have typically considered a small number of evaluation indicators or composition rules to form the relief layout. In this work, we integrate them to fully optimize the relief arrangement.

The evaluation indicators are divided into two categories based on the number of input models: one model versus multiple models. The used indicators for both categories are listed as below.

\section{A. Evaluation indicators for one model.}

$a_{1}$ : Projected area. This indicator is the projected area of the model in the relief. Introduced by [9], this measure was generated by the method mentioned in [10]. The graphical representation of this indicator is shown in Figure 3(a).

$a_{2}$ : Location of bounding rectangle. This indicator is summarized from photographic composition and the composition is defined as positioning the source figures appropriately to form a harmonious picture. Location of bounding rectangle is one of the most important rules in composition. Its value is equal to the Euclidean distance from the center of the source model to the center of the mapping region, which is expressed as dis. The graphical representation of this indicator is illustrated in Figure 3(b). When the center distance is reduced, the source model tends to move to the center of the mapping region.

$a_{3}$ : Curvature. A surface relief can be regarded as a complicated surface that attaches 3D models to a flat or curved target model. The source models deform along the surface. This indicator refers to the curvature of the source model after it is attached to the target model. In addition, the flatter the mapping region of the surface relief is, the smaller the deformation of the relief becomes. The graphical representation of this indicator is 


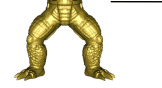

(a1) Input source object

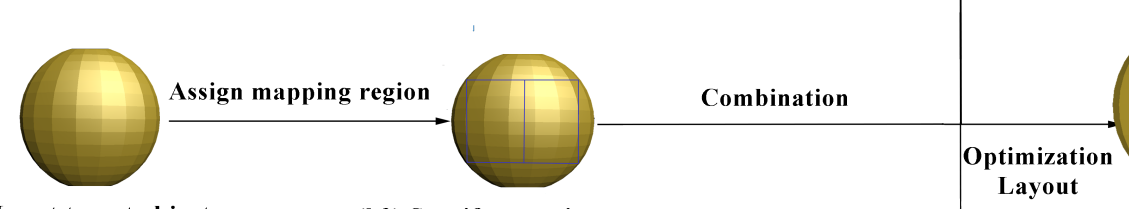

(b1) Input target object (b2) Specify mapping range

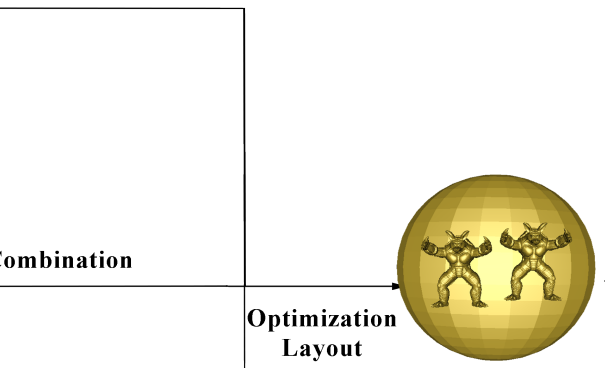

(d) Adjust Layout

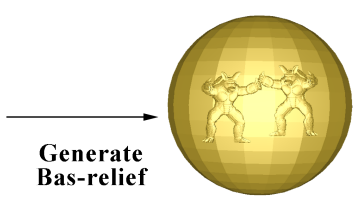

(e) The final result

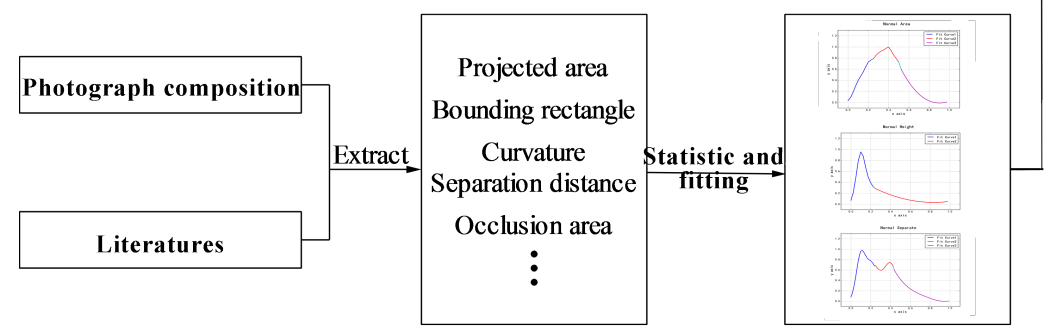

(c1) Preparation

(c2) Evaluation indicators

(c3) Fit goodness function

Figure 2: Overview of our approach.
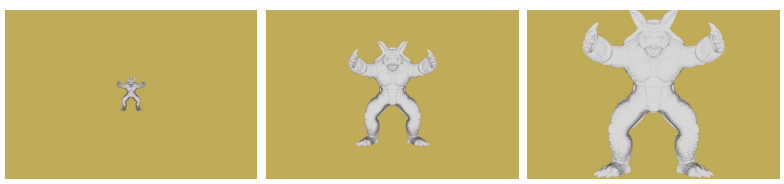

(a) Different values of projected area
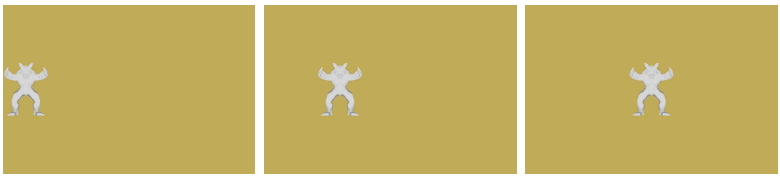

(b) Different values for the location of bounding rectangles

Figure 3: Schematic diagram of projected area and location of bounding rectangles.

displayed in Figure 4(a).

B. Evaluation indicators for multiple models.

$a_{4}$ : Separation distance. The distance between the source models affects the layout of the bas-relief. The separation distance could be too large or too small, which has a great impact on the aesthetic perception of the relief. The visualization of this indicator is shown in Figure 4(b).

$a_{5}$ : Occlusion area. If the separation distance between the source models is too small, the figures may be occluded. When the occlusion area between figures is too large, the figures cannot be clearly recognized. Thus, it is significant that we ensure the figures are visible.

$a_{6}$ : Height difference. We introduced this indicator
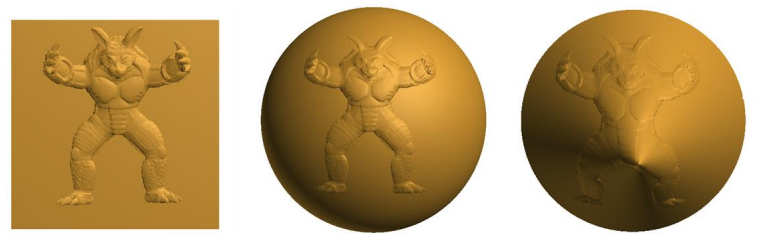

(a) Different values of curvature
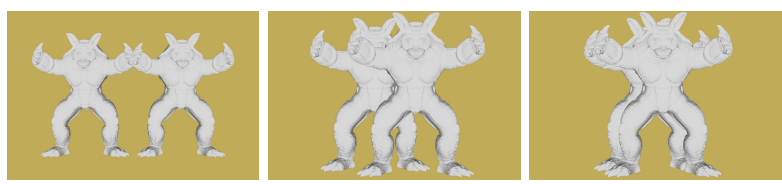

(b) Different values of separation distance

Figure 4: Results of curvature and separation distance.

to encourage a harmonious arrangement in the relief. The graphical representation of this indicator is shown in Figure 5(a).

$a_{7}$ : Symmetry. As one of the important relief layout indicators, we extend symmetry to our context of two models. Before we arrange two models in the mapping region, we roughly divide the target area into two parts (one for each model). The graphical representation of this indicator is shown in Figure 5(b).

\subsection{Evaluation Indicator Goodness Modeling}

Next we explain how to model the evaluation indicators which are collected by manual labeling and sta- 

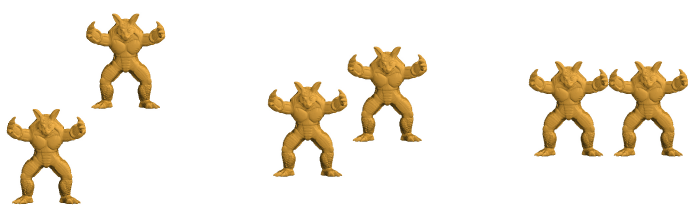

(a) Different values of height difference
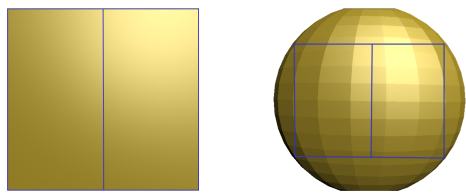

(b) Symmetry of mapping region

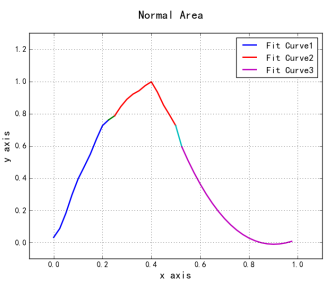

(a) Normalized Area

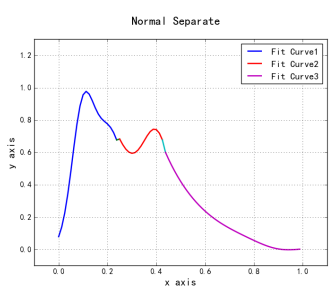

(c) Normalized Seperation

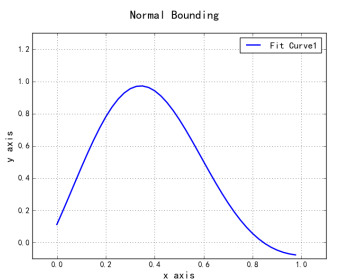

(b) Normalized Bounding

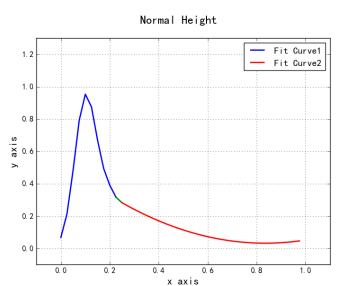

(d) Normalized Height
Figure 5: Results of height difference and symmetry.

tistical methods. To model these indicators, a number of issues arise. The first concern is the normalization of these evaluation indicators. It is difficult to train a model while the values of different indicators have different magnitudes. Second, we need to design a suitable function to quantify the output of these indicators.

We learn from classic relief pictures, extract the factors that influence the relief, and then build the evaluation indicators, as shown in Figure 6. Among them, the normalized area is the ratio of the area of the relief area in each image to the size of the input image. The normalized separation ratio is the boundary distance between the two reliefs in the image and the center of the two reliefs. The normalized height is the vertical direction of the two reliefs in the image to the relief height, and normalized bounding is the ratio of the distance from the center of the statistical relief to the center of the image. We adopt the Adaptive Mixed Gaussian function to fit the goodness functions of some evaluation indicators. Note that we simply set a fixed value for the projected area ratio indicator.

The adaptive performance is to determine whether the variance of the fitted function and the given curve is greater than a given threshold, thereby achieving a piece-wise Gaussian fitting. In the experiments, the parameters of the mixed Gaussian function are determined by the least squares curve fitting method, and it is defined in Equation (1). $\alpha_{1}, \alpha_{2}, \beta_{1}, \beta_{2}, \lambda_{1}, \lambda_{2}$ are the parameters to be determined.

$$
G(x)=\alpha_{1} \cdot e^{-\left(\frac{x-\beta_{1}}{\lambda_{1}}\right)^{2}}+\alpha_{2} \cdot e^{-\left(\frac{x-\beta_{2}}{\lambda_{2}}\right)^{2}}
$$

$a_{1}$ : Projected area. We assign a fixed value to $a_{1}$, which is equivalent to dividing the projected area of the mapping region by the sum of each projected area of the source models. Furthermore, Figure 6(a) shows that
Figure 6: Plots of the difference in each evaluation indicator in the user's preference. The horizontal axis is the normalized evaluation indicator in the range of 0 to 1 . The vertical axis is the percentage of the model layout based on the relief dataset and the current indicator value.

the surface relief of empirically best area ratio $a_{1}$ is 0.4 . When the $\mathrm{x}$-axis coordinate reaches 0.4 , the number of the bas-relief layout situation in the current area ratio reaches the maximum. This indicator does not participate in optimization calculation.

$a_{2}$ : Location of bounding rectangle. In the calculation process, we normalize the location of bounding rectangle by Eq. (2)(3)(4).

$$
\begin{gathered}
a_{2}=\operatorname{Euclidean}(\operatorname{median}(v(y))-\operatorname{median}(B(y)), \\
\text { median }(v(x))-\text { median }(B(x)))
\end{gathered}
$$

where $v(x)$ and $v(y)$ respectively denote the $x$-axis and $y$-axis coordinates of the source model. $B(x)$ and $B(y)$ denote the $\mathrm{x}$-axis and $\mathrm{y}$-axis coordinates of the mapping region, respectively.

Additionally,

$k_{2}=a_{2} /\left(a_{2}+\min ([\operatorname{median}(B(x))\right.$ median $\left.(B(y))]) / 2\right)$

$$
\begin{gathered}
N_{a_{2}}=1.59 * e^{-\left(\left(k_{2}-0.269\right) / 0.474\right)^{2}}- \\
1.56 * e^{-\left(\left(k_{2}-0.267\right) / 0.478\right)^{2}}
\end{gathered}
$$

where $k_{2}$ denotes the normalized value of $a_{2}$ and $N_{a_{2}}$ indicates the goodness function of $a_{2}$. With the least squares method to find the parameters of the fitting function, we get $\alpha_{1}=89.59, \alpha_{2}=-88.66, \beta_{1}=$ $0.269, \beta_{2}=0.267, \lambda_{1}=0.474, \lambda_{2}=0.478$. 
Table 1: Description of $\operatorname{llag}_{1}$.

\begin{tabular}{cc}
\hline flag $_{1}$ & intersections of two models \\
\hline $\mathbf{2}$ & intersect in $x$ axis but not intersect in $y$ \\
$\mathbf{1}$ & intersect in $y$ axis but not intersect in $x$ \\
$\mathbf{0}$ & intersect neither in $x$ axis nor in $y$ \\
$\mathbf{- 1}$ & intersect both in $y$ axis and $x$ \\
$\mathbf{- 2}$ & overlap \\
\hline
\end{tabular}

$a_{3}$ : Curvature. We employ the method [23] to calculate curvature $a_{3}$. The goodness function of $a_{3}$ is defined as

$$
N a_{3}=\sum[(\max (C)-C) /(\max (C)-\min (C))] / n
$$

where $C$ denotes the mean curvature of the source model which is already attached to the target model. $n$ denotes the number of vertices of the source model. When the target model is a complex surface, different layout positions will cause inconsistent curvatire calculations. Thereby, the Eq. (5) can reflect the object function.

a4: Separation distance. The layout will be affected by the difference in the horizontal or vertical distance between two source models. When the source model is attached to the target model, the $\mathrm{z}$-axis information needs to be recalculated, so we do not consider the $\mathrm{z}$ axis coordinate. Depending on the intersection situation, we define a piecewise function for five possible cases, which are shown in Figure 7.

$$
a_{4}=\left\{\begin{array}{ccc}
a b s\left(\operatorname { m a x } \left(\left[\min \left(v_{1}(y)\right)\right.\right.\right. & \left.\left.\min \left(v_{2}(y)\right)\right]\right)- & \\
\min \left(\left[\max \left(v_{1}(y)\right)\right.\right. & \left.\left.\left.\max \left(v_{2}(y)\right)\right]\right)\right) & \text { flag }_{1}=2 \\
a b s\left(\operatorname { m a x } \left(\left[\min \left(v_{1}(x)\right)\right.\right.\right. & \left.\left.\min \left(v_{2}(x)\right)\right]\right)- & \\
\min \left(\left[\max \left(v_{1}(x)\right)\right.\right. & \left.\left.\left.\max \left(v_{2}(x)\right)\right]\right)\right) & \text { flag }_{1}=1 \\
a b s\left(\operatorname { m a x } \left(\left[\min \left(v_{1}(y)\right)\right.\right.\right. & \left.\left.\min \left(v_{2}(y)\right)\right]\right)- & \\
\min \left(\left[\max \left(v_{1}(y)\right)\right.\right. & \left.\left.\left.\max \left(v_{2}(y)\right)\right]\right)\right) & \text { flag }_{1}=0 \\
-1, & & \text { flag }_{1}=-1 \\
-1, & & \text { flag }_{1}=-2
\end{array}\right.
$$

where $v_{1}(x)$ and $v_{1}(y)$ respectively denote the $x$-axis and $y$-axis coordinates of the first source model, and similarly $v_{2}(x)$ and $v_{2}(y)$ for the second source model. $a b s(x)$ is the absolute value of $x$ and flag $g_{1}$ indicates different layouts of the two models, shown in Table 1.

The normalized values and the goodness function are calculated by Eq. (7) and Eq. (8), respectively.

$k_{4}= \begin{cases}a_{4} / \operatorname{abs}\left(\text { median }\left(v_{1}(y)-\text { median }\left(v_{2}(y)\right)\right)\right. & \text { flag }_{1}=2 \\ a_{4} / \operatorname{abs}\left(\text { median }\left(v_{1}(x)-\text { median }\left(v_{2}(x)\right)\right)\right. & \text { flag }_{1}=1 \\ a_{4} / \operatorname{abs}\left(\text { median }\left(v_{1}(y)-\text { median }\left(v_{2}(y)\right)\right)\right. & \text { flag } g_{1}=0 \\ -1, & \text { flag }=-1 \\ -1, & \text { flag }=-2\end{cases}$

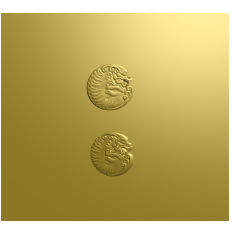

(a) $f \operatorname{lag}_{1}=2$

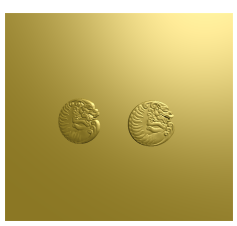

(b) flag $_{1}=1$

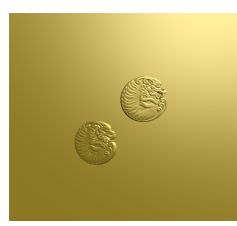

(c) $f \operatorname{lag}_{1}=0$

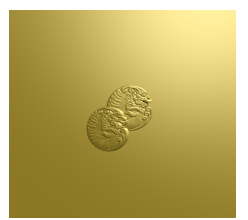

(d) flag $_{1}=-1$

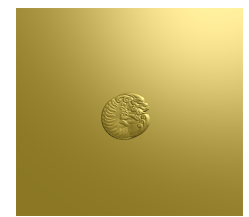

(e) flag $_{1}=-2$
Figure 7: Layouts with different separation distances.

$$
N_{a_{4}}= \begin{cases}0.736 * e^{-\left(\left(k_{4}-0.207\right) / 0.103\right)^{2}} & \\ +0.705 * e^{-\left(\left(k_{4}-0.096\right) / 0.062\right)^{2}} & k_{4} \leq 0.25 \\ 1.13 * e^{-\left(\left(k_{4}-0.066\right) / 0.252\right)^{2}} & \\ +0.551 * e^{-\left(\left(k_{4}-0.408\right) / 0.087\right)^{2}} & k_{4} \leq 0.45 \\ 5.06 * e^{-\left(\left(k_{4}-0.979\right) /-1.7\right)^{2}} & \\ -5.06 * e^{-\left(\left(k_{4}+1.19\right) / 493.39\right)^{2}} & k_{4} \leq 1\end{cases}
$$

where $k_{4}$ denotes the normalized value of $a_{4}$, the $f l a g_{1}$ parameters of Eq (8) are also shown in Table 1, and $N a_{4}$ denotes the goodness function of $a_{4}$. Note the goodness function is only valid when flag 1 is greater than -1 . According to different values of $k_{4}$, the parameters of $\alpha_{1}, \alpha_{2}, \beta_{1}, \beta_{2}, \lambda_{1}, \lambda_{2}$ will take corresponding values as shown in Eq. (8). Similarly, we adopt the least squares optimization to find the parameters of the fitting function. For instance, when $k_{4} \leq 0.25, \alpha_{1}=0.736$, $\alpha_{2}=0.705, \beta_{1}=0.207, \beta_{2}=0.096, \lambda_{1}=0.103$, $\lambda_{2}=0.062$.

$a_{5}$ : Occlusion area. We only consider the case in which the models are not occluded for simplicity. Hence, we define $f l a g_{2}$ as an identifier of whether one model occlude the other. When flag $_{2}$ equals to 1 , the models are not occluded, and occluded otherwise.

$$
N a_{5}= \begin{cases}1.0, & \text { flag }_{2}=1 \\ 0.0, & \text { flag }_{2}=0\end{cases}
$$

$a_{6}$ : Height difference. From photograph composition and literature, we found that the aesthetics of the relief are poor when two models are staggered. It can be formulated as:

$$
v_{1}(y)<v_{2}(y), o r, v_{2}(y)<v_{1}(y),
$$

The definition of the normalized values and height difference are shown in Eq. (12) and Eq. (13).

$a_{6}= \begin{cases}\operatorname{abs}\left(\text { median }\left(v_{1}(y)\right)-\text { median }\left(v_{2}(y)\right)\right) & \text { flag }_{3}=1 \\ 0, & \text { flag }_{3}=0\end{cases}$ 
where median $(x)$ denotes the median value of $x$ and $\mathrm{flag}_{3}$ is the identifier of whether the two models intersect in the longitudinal direction. When $\mathrm{flag}_{3}$ is equal to 1 , the models intersect, and they do not intersect otherwise.

$$
k_{6}=\left\{\begin{array}{cc}
a_{6} / \max \left[\max \left(v_{1}(y)\right)-\min \left(v_{1}(y)\right),\right. & \\
\left.\max \left(v_{2}(y)\right)-\min \left(v_{2}(y)\right)\right] & \text { flag }_{3}=1 \\
0, & \text { flag }_{3}=0
\end{array}\right.
$$

$$
N_{a_{6}}= \begin{cases}0.346 * e^{\left(-\left(\left(k_{6}-0.19\right) / 0.09\right)^{2}\right)} & \\ +0.82 * e^{\left(-\left(\left(k_{6}-0.097\right) / 0.06\right)^{2}\right)} & k \leq 0.25 \\ 1.3 * e^{\left(-\left(\left(k_{6}-0.105\right) / 0.46\right)^{2}\right)} & \\ -1.29 * e^{\left(-\left(\left(k_{6}-0.779\right) / 0.12\right)^{2}\right)} & k \leq 1\end{cases}
$$

Because the height difference is observed empirically, a subtle change of this indicator can have a great impact on the relief effects. We adopt adaptive Mixed Gaussian function to fit the goodness function of the height difference. Similarly, the values of $\alpha_{1}, \alpha_{2}, \beta_{1}, \beta_{2}$, $\lambda_{1}, \lambda_{2}$ are shown in Eq. (13).

$a_{7}$ : Symmetry. Since we segment the mapping region into two parts along the medial axis, the mapping region for each model changes, as shown in Eq. (14). According to Eq. (14), we constraint different models in the left and right positions of the middle axis.

$$
\begin{gathered}
B^{\text {left }}\left(x_{1}\right)_{\max }=\max (B(x))- \\
(\max (B(x))-\min (B(x)) / 2), \\
B^{\text {left }}\left(x_{2}\right)_{\min }=\min (B(x))+ \\
\quad(\max (B(x))-\min (B(x)) / 2),
\end{gathered}
$$

where $B(x)$ denotes the bounding box of the model, which contains $\left(x_{\min }, x_{\max }, y_{\min }, y_{\max }\right)$ coordinates. The change of this parameter will affect the variables depending on the location of bounding rectangle.

\section{Relief Layout Optimization}

Our relief layout problem is formulated as a combinatorial optimization problem: a combination of all items from the list of candidate indicators are found to best satisfy both the photographic composition and aesthetics rules. To quickly obtain a suitable solution, we employ a classical heuristic search algorithm, simulated annealing which is commonly used in furniture arrangement [18] and building layout planning [24]. We first define the objective function in the heuristic process using the evaluation indicators, and then construct the simulated annealing model.

\section{1 objective Function}

We denote the $i$-th source model by $S_{i}$, and $M=$ $\left\{\left(S_{i}, a_{j}\right), 1 \leq i \leq n, 1 \leq j \leq m\right\}$ denotes the con- figuration of the relief layout, where $n$ is the number of source models, $a_{j}$ is the $j$-th evaluation indicator of the $i$-th source model, and $m$ denotes the number of evaluation indicators. Our objective function for the layout configuration is divided into two categories according to the number of source models. For different layout strategies, we propose different objective functions to solve the problem. As shown in Eq. (15), for one source model the objective function consists of two terms; while for two models as Eq. (16), the objective function consists of five terms. At the same time, the change of the objective function state needs to be within the scope of the relief area.

$$
C(M)=\left(N a_{2}\right)^{w_{2}} *\left(N a_{3}\right)^{w_{3}},
$$

$$
C(M)=\prod_{j}^{m} \prod_{i}^{n}\left(N a_{j}{ }^{i}\right)^{w_{j}^{i}}
$$

where $w_{j}^{i}$ is the weight for the $j$-th goodness of evaluation indicator of the $i$-th model. The $w$ coefficients determine the relative weighting between the objective terms; we empirically set $w_{2}=5.0, w_{3}=1.0$ in Eq. (15), and $w_{2}^{i}=1.0, w_{3}^{i}=1.0, w_{4}^{i}=2.0$, and $w_{6}^{i}=1.0$ in Eq. (16).

\subsection{Simulated Annealing}

The goal of simulated annealing is to find the best relief layout that combines the evaluation indicators mentioned above (Sec 4.1. In a typical simulated annealing process, the initial configuration $M_{0}$ is generated by randomly assigning a position to each source model in the relief. In the $k$-th iteration, we find a new configuration $M_{k}^{\prime}$ in the neighborhood of $M_{k} . M_{k}^{\prime}$ is accepted as $M_{k+1}$ with probability $p_{r}, T_{0}$ indicates te initial temperature, $\delta$ is the decrease ratio in temperature during each iteration, which is defined by Eq. (17),Eq. (18).

$$
\begin{gathered}
\operatorname{pr}_{r}\left(M_{k}^{\prime} \rightarrow M_{k+1}\right)= \\
\min \left\{1, \exp \left[-\left(C\left(M_{k}^{\prime}\right)-c\left(M_{k}\right)\right) /(T(k))\right]\right\} \\
T(K)=\delta \frac{T_{K-1}}{\log (1+K)}
\end{gathered}
$$

In this study, we empirically set $\delta=0.95, T_{0}=1.5$ to initialize the simulated annealing algorithm.

Neighborhood. The neighborhood of a configuration indicates the potential movement at each iteration. At each iteration, we randomly choose one of the four directions (up, down, left, right), and change its current position to another.

\section{Results}

To demonstrate the effectiveness of our method, we employ [25] to generate bas-relief based on our layout results. All the experiments are implemented on a desktop 

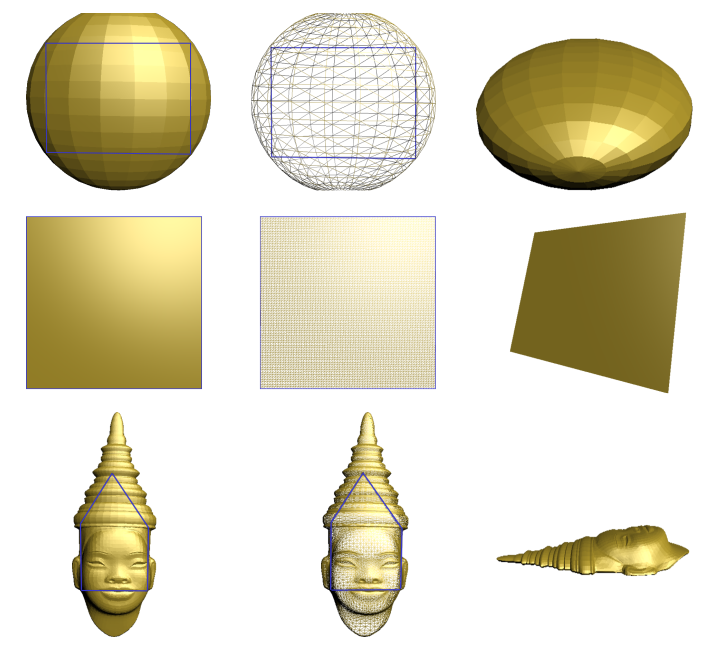

Figure 8: The target models. The regions surrounded by blue lines are the mapping regions. First column: the models with flat line shader. Second column: the models with wireframe shader. Third column: the side view of the models.

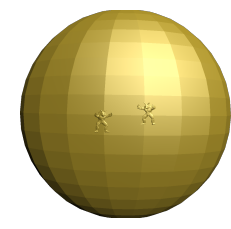

(a)

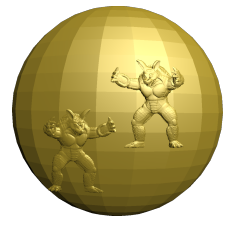

(b)

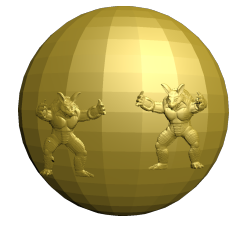

(c)
Figure 9: Layout results without certain evaluation indicators.

PC with an Intel Core i5-7300HQ CPU and 16.0GB of RAM. Figure 8 shows the target objects we used in our experiments.

The primary goal of the experiments is to validate the quality of the relief layout synthesized using our approach. Our experimental procedure is mainly divided into two phases. In the first phase, we disable some of the evaluation indicators and use linear function as the objective function, given by Eq. (19). Figure 9 shows the results of preliminary experiments, which use the linear model as the objective function.

$$
C(M)=\sum_{i=1}^{n} N a_{i},
$$

Improvement. The results (Figure 9) of the first phase are not decent. As we can see, without considering the height difference, the layout of the source models is unbalanced and incongruous. Additionally, if the projection area of the source model is not fixed, it takes much more time to calculate and the results are unstable.

Figure 9(a) shows the results without considering the projected area. In this case, the occupied area of basrelief subjects is very small, whereas the base model

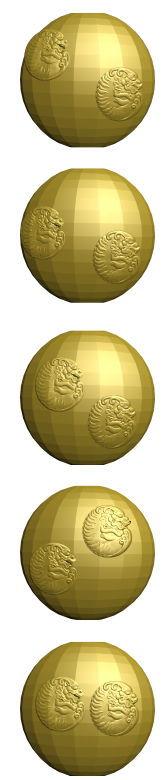

(a)
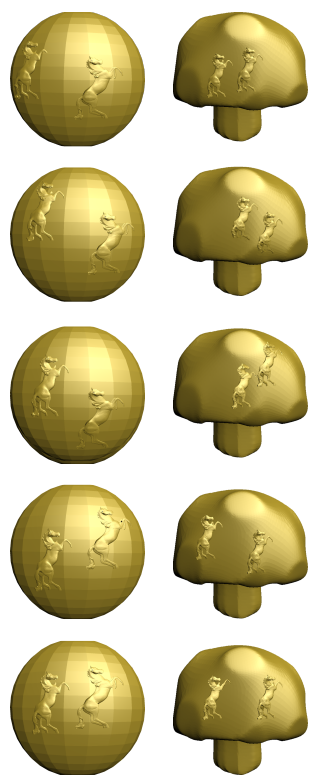

(b)

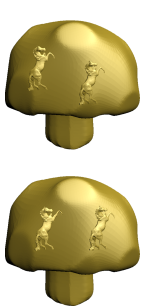

(c)
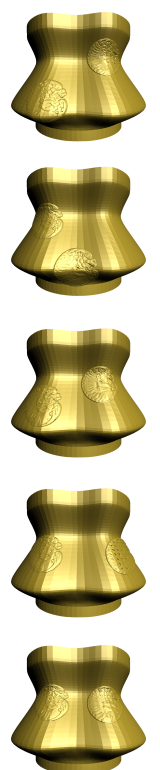

(d)

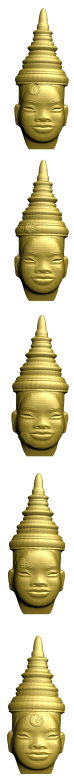

(e)
Figure 10: Our results.

gains the most attention. Figure 9(b) represents the results without considering the height difference. As the figure shows, the bas-relief layout could be arranged symmetrically. By contrast, because of the height difference between them, the bas-reliefs were not as arranged as we expected. Figure 9(c) presents the results without considering the separation distance. Therefore, the spacing of the model layout is too large.

Performance. As shown in Figure 10, our approach can automatically place the input source models into the mapping regions. Figure 10(a) and Figure 10(b) show the layout of two models arranged on a sphere surface model. Figure 10(c) and Figure 10(d) show the layout of the two models arranged on a complex target model. Figure 10(e) shows the layout of one model arranged on a mask model. The first four rows represent the initial configurations of the relief layouts, which are generated using a random value of the evaluation indicators. The last row represents the final results of the relief layouts.

Comparison. To illustrate the effectiveness of the Bas-relief layout method, we compare the experimental results of the layout with the results from [26] which creates the appearance-mimicking surface from the artificially designed 3D scene. This method [26] requires manual layout of the 3D scene models, which is timeconsuming and unable to adapt to changing art trends. Our method only needs to provide the source model and target model, so that it can efficiently generate a basrelief, as shown in the Figure 11(b). Figure 11 show that the result of [26] has obvious overlap in layout, while our method enables a layout according to the defined 
indicators.

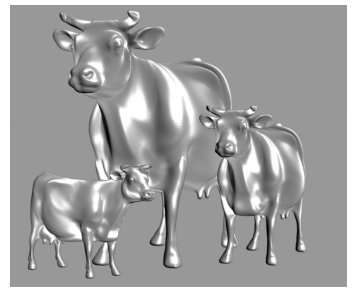

(a)

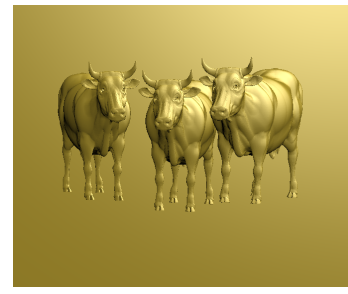

(b)
Figure 11: Comparison. (a) Bas-relief layout created by [26]. (b) Bas-relief layout generated using our method.

User survey. We evaluate the bas-relief layout images generated by our method on the online crowdsourcing platform in mainland China (called questionnaire star), which provides functions equivalent to Amazon Mechanical Turk platform. We invited 60 undergraduate students to participate in the following questionnaire, which involved 7 cases in Figure 10. In each case, the participants evaluate 5 images of the bas-relief layout, including an image of the initial random layout of the bas-relief model, 3 layout images output during the optimization process, and a final model layout image. In the same case, six images are presented to the participants in a random order and they are requested to rate each image from a scale of 1-5. A higher score indicates a higher level of the bas-relief model conforming to aesthetics. Figure 12 counts the average score of 60 participants for each case, the statistical result shows that our results outperform the initial layout. We can see that our method enables 3 cases with the highest score in 7 cases $(42.9 \%=3 / 7)$, and 6 cases with the top two scores $(85.7 \%=6 / 7)$, which demonstrates the effectiveness of our approach.

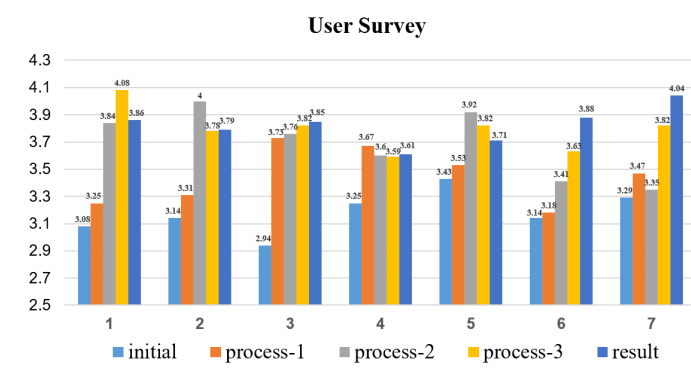

Figure 12: Statistics of our user survey. The different colors represent the average evaluation scores for the bas-relief layouts by initial random configuration, optimization process and the final result.

\section{Conclusion and Future work}

This paper presents a novel bas-relief layout generation method. To build the objective function, we extract evaluation indicators of the arrangement and assembled into a function. To deal with the prohibitively large calculation, the objective function is optimized by simulated annealtion. Experiments have demonstrated that our method can produce robust and aesthetic results.

One main limitation exists. It is currently suitable for arranging a limited number of source models. Complexity will significantly increase with the growing number of source objects. In the future, we would like to explore this research point.

\section{References}

[1] Yu-Wei Zhang, Caiming Zhang, Wenping Wang, Yanzhao Chen, Zhongping Ji, and Liu Hui. Portrait relief modeling from a single image. IEEE transactions on visualization and computer graphics, 2019.

[2] Yu, Wei Zhang, Jing Wu, Zhongping Ji, Mingqiang Wei, and Caiming Zhang. Computer assisted relief modelling: A comprehensive survey. Computer graphics forum: journal of the European Association for Computer Graphics, 2019.

[3] Yuwei. Zhang, Yiqi. Zhou, Xiaofeng. Zhao, and Gang. Yu. Real-time bas-relief generation from a 3d mesh. Graphical Models graphical Models and Image Processing computer Vision, Graphics, and Image Processing, 75(1):2-9, 2013.

[4] Zhongping Ji, Weiyin Ma, and Xianfang Sun. Basrelief modeling from normal images with intuitive styles. IEEE transactions on visualization and computer graphics, 20(5):675-685, 2013.

[5] Zhongping. Ji, Xianfang. Sun, Shi. Li, and Yigang. Wang. Real-time bas-relief generation from depth-and-normal maps on gpu. symposium on geometry processing, 33(5):75-83, 2014.

[6] Meili Wang A, Li Wang B, Tao Jiang B, Nan Xiang $\mathrm{B}$, Juncong Lin E, Mingqiang Wei $\mathrm{C}$, Xiaosong Yang B, Taku Komura D, and Jianjun Zhang B. Bas-relief modelling from enriched detail and geometry with deep normal transfer sciencedirect. Neurocomputing, 2020.

[7] Jianping. Hu, Xiaochao. Wang, and Hong. Qin. Improved, feature-centric emd for $3 \mathrm{~d}$ surface modeling and processing. Graphical Models graphical 
Models and Image Processing computer Vision, Graphics, and Image Processing, 76(5):340-354, 2014.

[8] Dongbo. Zhang, Xiaochao. Wang, Jianping. Hu, and Hong. Qin. Interactive modeling of complex geometric details based on empirical mode decomposition for multi-scale $3 \mathrm{~d}$ shapes. Computeraided Design, 87:1-10, 2017.

[9] Jingqiao. Hu, Sha. He, and Lyu. Lin. Placement optimization for generating bas-reliefs based on visual saliency. Journal of Computer-Aided Design \& Computer Graphics, 28(12):2128-2133, 2016.

[10] Tingting Li, Liying Yang, Meili Wang, Yuling Fan, Feiyu Zhang, Shihui Guo, Jian Chang, and Jian Jun Zhang. Visual saliency-based basrelief generation with symmetry composition rule. Computer Animation and Virtual Worlds, 29(34):e1815, 2018.

[11] Zhongping. Ji, Xianfang. Sun, and Weiyin. Ma. Normal image manipulation for bas-relief generation with hybrid styles. arXiv: Graphics, 2018.

[12] Zhongping Ji, Wei Feng, Xianfang Sun, Feiwei Qin, and Weiyin Ma. Reliefnet: Fast bas-relief generation from 3d scenes. Computer-Aided Design, 130:102928, 2020.

[13] Song Hai Zhang, Shao Kui Zhang, Yuan Liang, and Peter Hall. A survey of 3d indoor scene synthesis. Journal of Computer Science and Technology, 34(3):594-608, 2019.

[14] Matthew Fisher and Pat Hanrahan. Context-based search for 3d models. In Acm Siggraph Asia, pages $1-10,2010$.

[15] Matthew. Fisher, Manolis. Savva, Yangyan. Li, and Pat. Hanrahan. Activity-centric scene synthesis for functional $3 \mathrm{~d}$ scene modeling. Acm Transactions on Graphics, 34(6):179, 2015.

[16] Manolis. Savva, Angel X. Chang, Pat. Hanrahan, and Matthew. Fisher. Pigraphs: learning interaction snapshots from observations. Acm Transactions on Graphics, 35(4):139, 2016.

[17] Paul. Merrell, Eric. Schkufza, Zeyang. Li, Maneesh. Agrawala, and Vladlen. Koltun. Interactive furniture layout using interior design guidelines. ACM Transactions on Graphics (TOG), 30(4):110, 2011.
[18] Lap Fai. Yu, Sai Kit. Yeung, Chi Keung. Tang, Demetri. Terzopoulos, Tony F. Chan, and Stanley J. Osher. Make it home:automatic optimization of furniture arrangement. Acm Transactions on Graphics, 30(4):1-12, 2011.

[19] Qiang. Fu, Xiaowu. Chen, Xiaotian. Wang, Sijia. Wen, Bin. Zhou, and Hongbo. Fu. Adaptive synthesis of indoor scenes via activity-associated object relation graphs. international conference on computer graphics and interactive techniques, 36(6):201, 2017.

[20] Peter. Kan and Hannes. Kaufmann. Automated interior design using a genetic algorithm. page 25 , 2017.

[21] Yang Yang, Shi Jin, Ruiyang Liu, Sing Bing Kang, and Jingyi Yu. Automatic 3d indoor scene modeling from single panorama. In 2018 IEEE/CVF Conference on Computer Vision and Pattern Recognition (CVPR), 2018.

[22] Peter JM Van Laarhoven and Emile HL Aarts. Simulated annealing. In Simulated annealing: Theory and applications, pages 7-15. Springer, 1987.

[23] David. Cohen-Steiner and Jean .Marie. Morvan. Restricted delaunay triangulations and normal cycle. In Nineteenth Symposium on Computational Geometry, pages 312-321, 2003.

[24] Paul. Merrell, Eric. Schkufza, and Vladlen. Koltun. Computer-generated residential building layouts. Acm Transactions on Graphics, 29(6):112, 2010.

[25] Tingting, Yang, Liying, Wang, Meili, Fan, Yuling, Zhang, Feiyu, and Guo and. Visual saliencybased bas-relief generation with symmetry composition rule. Computer animation and virtual worlds, 2018.

[26] Christian Schuller, Daniele Panozzo, and Olga Sorkine-H. Appearance mimicking surfaces. Acm Transactions on Graphics, 33(6):216:1-216:10, 2014. 